\title{
LETTER
}

\section{Organizational aspects of care associated with mortality in critically ill COVID-19 patients}

\author{
Thomas Rimmelé ${ }^{1,2}$, Léa Pascal ${ }^{3,4}$, Stéphanie Polazzi ${ }^{3,4}$ and Antoine Duclos ${ }^{3,4^{*}}$ (0)
}

๑) 2020 Springer-Verlag GmbH Germany, part of Springer Nature

\section{Dear Editor,}

The coronavirus disease 2019 (COVID-19) pandemic has challenged hospital organizations worldwide, not only because of the novelty of the disease, but also because of the high volume of patients in need of critical care over a short time period [1]. ICU mortality of COVID-19 patients depends on patient-related and caregiver-related factors in addition to organizational aspects of the unit, where those patients are hospitalized. We sought to identify various organizational factors associated with ICU mortality among COVID-19 patients.

We performed a nationwide study based on the medical information system from all public and private hospitals in France. All adults admitted to a French ICU for severe COVID-19 acute respiratory failure, with SAPS II greater than 15 and who received invasive ventilation, between January 1, 2020, and April 26, 2020 were included. The primary outcome was all-cause mortality during the ICU stay. We computed a modified Poisson regression model to estimate the influence on patient mortality of organizational factors including a potential weekend effect (death probability among patients discharged from ICU on Saturday or Sunday compared to other weekdays), hospital location in French regions, and ICU team experience over time (cumulative number of COVID-19 patients already admitted to the ICU) [2].

A total of 9809 patients from 350 hospitals were analyzed, with a median of 17 severe COVID-19 patients (range 1-230) and 4 related deaths (0-97) per ICU. Patients mean age was 63.2 years (SD 11.6), SAPS II was 45.4 (16.9) and ICU length of stay 20.5 days (16.1). Overall, 3069 (31.3\%) patients died in ICU. After adjusting for patient-related confounders, the risk of death increased among weekend ICU discharges (relative Risk 1.54, 95\% CI 1.45-1.64). Patient mortality was also higher within ICUs located in the Paris $(1.62,1.35-1.94)$ and Northeast $(1.24,1.02-1.49)$ regions (Table 1$)$.

Three findings result from this large data analysis limited to available medical information that may not always consider all possible confounders accurately. First, weekends were associated with an increased likelihood of patient death at the end of ICU stay. Understaffing frequently occurs during weekends [3] and this result can be interpreted as a lack of available health professionals, given the patients' needs [4]. Second, excess mortality may arise when healthcare organizations are overwhelmed. Paris and Northeast regions exhibited by far the highest number of severe COVID-19 patients to treat in France and corresponding ICUs appeared to be rapidly saturated [5]. Finally, no learning curve for ICU management of COVID-19 patients was evidenced. A potential explanation is that "practice makes perfect" effect may be counterbalanced by high-volume of admissions leading to excessive workload and surpassing bed capacity to provide optimal care.

In the aftermath of the COVID-19 pandemic, ICU organizational aspects significantly influenced patient outcome. The capacity of healthcare systems to reshape quickly seems crucial to population survival in the context of health crises. Solutions to avoid overwhelming situations may include appropriate staffing, temporary units' openings, and close collaborations between ICUs from the same territory for optimal patient repartition.

\footnotetext{
*Correspondence: antoineduclos@yahoo.fr

${ }^{3}$ Health Data Department, Hospices Civils de Lyon, Lyon, France

Full author information is available at the end of the article
}

\section{Springer}


Table 1 Factors associated with ICU mortality among COVID-19 patients

\begin{tabular}{|c|c|c|c|c|}
\hline \multirow[b]{2}{*}{ Factors } & \multicolumn{2}{|l|}{ Unadjusted } & \multicolumn{2}{|l|}{ Adjusted } \\
\hline & Relative risks $(95 \% \mathrm{Cl})$ & $p$ value & Relative risks $(95 \% \mathrm{Cl})$ & $p$ value \\
\hline \multicolumn{5}{|l|}{ Day of ICU discharge } \\
\hline Weekend & $1.65(1.54-1.77)$ & $<0.001$ & $1.54(1.45-1.64)$ & $<0.001$ \\
\hline Other weekdays & 1 & Reference & 1 & Reference \\
\hline \multicolumn{5}{|l|}{ ICU location in France } \\
\hline Paris region & $1.59(1.3-1.95)$ & $<0.001$ & $1.62(1.35-1.94)$ & $<0.001$ \\
\hline Northeast & $1.35(1.1-1.68)$ & 0.005 & $1.24(1.02-1.49)$ & 0.029 \\
\hline Northwest & $1.07(0.83-1.37)$ & 0.604 & $1.14(0.93-1.4)$ & 0.194 \\
\hline Southeast & $1.28(1.03-1.58)$ & 0.024 & $1.11(0.93-1.33)$ & 0.258 \\
\hline Southwest & 1 & Reference & 1 & Reference \\
\hline \multicolumn{5}{|c|}{ ICU team experience over time $e^{a}$} \\
\hline Very high [44-229 patients] & $0.82(0.74-0.9)$ & $<0.001$ & $0.97(0.86-1.1)$ & 0.664 \\
\hline High [20-43 patients] & $0.86(0.79-0.94)$ & 0.001 & $0.98(0.9-1.07)$ & 0.661 \\
\hline Low [8-19 patients] & $0.88(0.81-0.96)$ & 0.004 & $0.94(0.87-1.02)$ & 0.147 \\
\hline Very low [0-7 patients] & 1 & Reference & 1 & Reference \\
\hline \multicolumn{5}{|l|}{ Patient ICU admission date } \\
\hline April 13 to April 26 & $0.88(0.76-1.02)$ & 0.092 & $1.14(0.97-1.35)$ & 0.113 \\
\hline March 30 to April 12 & $0.72(0.65-0.8)$ & $<0.001$ & $1.01(0.89-1.15)$ & 0.873 \\
\hline March 16 to March 29 & $0.81(0.73-0.9)$ & $<0.001$ & $1.08(0.97-1.2)$ & 0.164 \\
\hline January 01 to March 15 & 1 & Reference & 1 & Reference \\
\hline \multicolumn{5}{|l|}{ Patient sex } \\
\hline Male & $1.06(0.99-1.13)$ & 0.080 & $1.04(0.98-1.09)$ & 0.229 \\
\hline Female & 1 & Reference & 1 & Reference \\
\hline \multicolumn{5}{|l|}{ Patient age, year } \\
\hline $80+$ & $5.38(3.62-8)$ & $<0.001$ & $3.92(2.96-5.2)$ & $<0.001$ \\
\hline $75-79$ & $3.91(2.64-5.78)$ & $<0.001$ & $2.77(2.11-3.64)$ & $<0.001$ \\
\hline $70-74$ & $2.96(2.01-4.35)$ & $<0.001$ & $2.12(1.61-2.78)$ & $<0.001$ \\
\hline $60-69$ & $2.36(1.6-3.48)$ & $<0.001$ & $1.78(1.37-2.3)$ & $<0.001$ \\
\hline $40-59$ & $1.34(0.92-1.95)$ & 0.127 & $1.17(0.91-1.51)$ & 0.218 \\
\hline 18-39 & 1 & Reference & 1 & Reference \\
\hline \multicolumn{5}{|l|}{ Patient SAPS II ${ }^{a}$} \\
\hline Very high [56-120] & $3.03(2.66-3.44)$ & $<0.001$ & $1.79(1.6-2.01)$ & $<0.001$ \\
\hline High [43-55] & $2.12(1.87-2.4)$ & $<0.001$ & $1.39(1.25-1.55)$ & $<0.001$ \\
\hline Low [33-42] & $1.65(1.46-1.88)$ & $<0.001$ & $1.27(1.13-1.42)$ & $<0.001$ \\
\hline Very low [15-32] & 1 & Reference & 1 & Reference \\
\hline \multicolumn{5}{|l|}{ Charlson comorbidity index } \\
\hline $3+$ & $1.36(1.36-1.51)$ & $<0.001$ & $1.03(0.94-1.13)$ & 0.553 \\
\hline 2 & $1.15(1.15-1.27)$ & 0.010 & $0.96(0.89-1.05)$ & 0.403 \\
\hline 1 & $1.3(1.17-1.43)$ & $<0.001$ & $1.07(0.97-1.18)$ & 0.179 \\
\hline 0 & 1 & Reference & 1 & Reference \\
\hline \multicolumn{5}{|l|}{ Hemodynamic support } \\
\hline Yes & $2.1(1.84-2.4)$ & $<0.001$ & $1.60(1.42-1.8)$ & $<0.001$ \\
\hline No & 1 & Reference & 1 & Reference \\
\hline \multicolumn{5}{|l|}{ Renal replacement therapy } \\
\hline Yes & $2.23(2.07-2.4)$ & $<0.001$ & $1.84(1.72-1.97)$ & $<0.001$ \\
\hline No & 1 & Reference & 1 & Reference \\
\hline \multicolumn{5}{|c|}{ Patient median household income ${ }^{a}, \epsilon$} \\
\hline Very low $[11,726-18,115]$ & $1.19(1.1-1.28)$ & $<0.001$ & $1.23(1.14-1.33)$ & $<0.001$ \\
\hline Low $[18,125-20,083]$ & $1.11(1.01-1.22)$ & 0.025 & $1.12(1.03-1.22)$ & 0.009 \\
\hline High $[20,083-22,582]$ & $1.08(0.99-1.18)$ & 0.094 & $1.11(1.03-1.2)$ & 0.009 \\
\hline Very high $[22,583-43,350]$ & 1 & Reference & 1 & Reference \\
\hline
\end{tabular}


Table 1 (continued)

9809 critically ill COVID-19 patients from 350 hospitals were analyzed. Using modified Poisson regression model (with a robust error variance) accounting for patient clustering within hospitals and for patient related confounders (sex, age, SAPS II, Charlson comorbidity index, hemodynamic support, renal replacement therapy, patient median household income) and the date of patient ICU admission, we estimated adjusted relative risks with their $95 \%$ confidence intervals (95\% CI)

a Categorized into quartiles

\section{Author details}

${ }^{1}$ Anesthesiology and Critical Care Medicine, Edouard Herriot Hospital, Hospices Civils de Lyon, Lyon, France. ${ }^{2}$ Pathophysiology of Injury-Induced Immunosuppression (Pi3, EA 7426), Université Claude Bernard Lyon 1, Lyon, France. ${ }^{3}$ Health Data Department, Hospices Civils de Lyon, Lyon, France. ${ }^{4}$ Health Services and Performance Research Lab (HeSPeR, EA 7425), Université Claude Bernard Lyon 1, Lyon, France.

\section{Author contributions}

$A D, L P, S P$, and TR prepared this paper; AD and TR collaborated in the concept and design of the study; $A D$ and TR was responsible for study governance and logistical support; all authors contributed substantially to analyzing and interpretation of data; $A D$ and TR drafted the manuscript which then was critically revised by all the authors; All authors have read and approved the final manuscript.

\section{Funding}

None declared.

\section{Compliance with ethical standards}

\section{Conflicts of interest}

All authors declare no conflicts of interest.

\section{Ethical approval}

This observational study was based on anonymous data and declared to the National Data Protection Commission (MR-4423250520) before any analysis.
Accepted: 15 September 2020

Published online: 6 October 2020

\section{References}

1. Aziz S, Arabi YM, Alhazzani W, Evans L, Citerio G, Fischkoff K, Salluh J, Meyfroidt G, Alshamsi F, Oczkowski S, Azoulay E, Price A, Burry L, Dzierba A, Benintende A, Morgan J, Grasselli G, Rhodes A, Moller MH, Chu L, Schwedhelm S, Lowe JJ, Bin D, Christian MD (2020) Managing ICU surge during the COVID-19 crisis: rapid guidelines. Intensive Care Med 46(7):1303-1325. https://doi.org/10.1007/s00134-020-06092-5

2. Zou G, Donner A (2013) Extension of the modified Poisson regression model to prospective studies with correlated binary data. Stat Methods Med Res 22(6):661-670. https://doi.org/10.1177/0962280211427759

3. Neuraz A, Guérin C, Payet C, Polazzi S, Aubrun F, Dailler F, Lehot JJ, Piriou V, Neidecker J, Rimmelé T, Schott AM, Duclos A (2015) Patient mortality is associated with staff resources and workload in the ICU: a multicenter observational study. Crit Care Med 43(8):1587-1594. https://doi. org/10.1097/CCM.0000000000001015

4. Honeyford K, Cecil E, Lo M, Bottle A, Aylin P (2018) The weekend effect: does hospital mortality differ by day of the week? A systematic review and meta-analysis. BMC Health Serv Res 18(1):870. https://doi. org/10.1186/s12913-018-3688-3

5. COVID19-APHP Group (2020) Assistance Publique-Hôpitaux de Paris' response to the COVID-19 pandemic. Lancet 395(10239):1760-1761. https://doi.org/10.1016/S0140-6736(20)31210-1

\section{Publisher's Note}

Springer Nature remains neutral with regard to jurisdictional claims in published maps and institutional affiliations. 